\title{
Factores de riesgo en la mortalidad de los recién nacidos de muy bajo peso con membrana hialina
}

\author{
M.B. García Ariasa, P. Zuluaga Arias ${ }^{\mathrm{b}}, \mathrm{M}^{\mathrm{a}}$ C. Arrabal Terán ${ }^{\mathrm{c}}$ y J. Arizcun Pineda ${ }^{\mathrm{c}}$
}

${ }^{a}$ Centro de Salud Cerro Almodóvar. ${ }^{b}$ Departamento de Estadística e IOI. Facultad de Medicina. Universidad Complutense. 'Servicio de Neonatología. Hospital Clínico San Carlos. Madrid. España.

\section{Introducción}

La expectativa de vivir de los recién nacidos de muy bajo peso (RNMBP) ha mejorado en los últimos años. Cuando estos pacientes presentan, además, enfermedad de membrana hialina $(\mathrm{EMH})$, es difícil conocer con seguridad cuáles son los factores que más influyen en su mortalidad. Este trabajo tiene el objetivo de averiguar, dentro de un conjunto de variables, las más influyentes en la mortalidad desde una perspectiva multifactorial.

\section{Pacientes y métodos}

Se tomó una muestra de 209 RNMBP con EMH nacidos en un período amplio, 15 años y 7 meses. Se consideraron las variables: "fecha de parto", "grado de enfermedad de membrana hialina", "sexo", "peso al nacimiento", "semanas de gestación", "procedencia", "administración de corticoides prenatales", "tipo de gestación", "tipo de parto", "momento de la amniorrexis", "puntuación del test de Apgar al minuto y a los 5 min", "administración de surfactante", "horas de vida en la administración de la primera dosis de surfactante" y "sepsis precoz". Siguiendo la metodología de selección de variables de HosmerLemeshow se realizó un análisis de regresión logística múltiple.

\section{Resultados}

Resultaron significativas las variables peso al nacimiento; test de Apgar, 5; corticoides prenatales, grado de membrana hialina y tratamiento con agente tensioactivo, quedando la importancia del resto de las variables diluida en ellas.

\section{Conclusiones}

El incremento de peso y el test de Apgar a los $5 \mathrm{~min}$, la administración de agente tensioactivo y corticoides, así como un grado bajo de membrana hialina hacen que la mortalidad disminuya. El modelo de regresión logística encontrado cuantifica cómo actúan estos factores y permite estimar la probabilidad de fallecer para un nuevo RNMBP con EMH.

\section{Palabras clave:}

Recién nacido de muy bajo peso. Enfermedad de membrana hialina. Regresión logística. Mortalidad.

\section{RISK FACTORS FOR MORTALTY IN VERY LOW BIRTH WEGHT INFANTSWITH RESPIRATORY DISTRESS SYNDROME}

\section{Introduction}

In the last few years the life expectancy of very low birth weight (VLBW) infants has improved. When these patients have respiratory distress syndrome, it is difficult to know with any certainty which factors have the greatest influence on mortality. The aim of this study was to determine which variables, among a series, have the greatest influence on mortality from a multivariate perspective.

\section{Patients and methods}

A sample of 209 VLBW infants born over a long period (15 years and 7 months) was studied. The following variables were analyzed: date of birth, degree of respiratory distress syndrome, sex, birth weight, weeks of gestation, born within or elsewhere, prenatal corticoid administration, type of gestation, type of delivery, amniorrhexis time, Apgar test at 1 and 5 minutes, surfactant administration, hours of life at which the first dose of surfactant was administered, and early sepsis. A multiple logistic regression analysis was developed using Hosmer-Lemeshow methodology.

Correspondencia: Dra. M.B. García Arias.

Centro de Salud Cerro Almodóvar.

Fuentespina, s/n. 28031 Madrid. España.

Correo electrónico: martagarias@telefonica.net

Recibido en septiembre de 2004.

Aceptado para su publicación en mayo de 2005. 


\section{Results}

The following variables were identified as significant: birth weight, Apgar test at 5 minutes, prenatal corticoids, degree of respiratory distress syndrome, and surfactant administration. The remaining variables were less important in the multivariate analysis.

\section{Conclusions}

Higher birth weight and Apgar score at 5 minutes, prenatal corticoid and surfactant administration, and a lower degree of respiratory distress syndrome reduce mortality. The logistic regression model used quantifies how these factors behave and allows the probability of mortality in VLBW infants with respiratory distress syndrome to be estimated.

\section{Key words:}

Very low birth weight infant. Respiratory distress syndrome. Logistic regression. Mortality.

\section{INTRODUCCIÓN}

El recién nacido de muy bajo peso (RNMBP), con peso igual o inferior a $1.500 \mathrm{~g}$, por sus peculiaridades anatómicas y fisiológicas, ha planteado siempre un importante motivo de debate y discusión, respecto a cuestiones como a partir de qué peso o edad gestacional considerar su viabilidad y comenzar una reanimación. Un trastorno que presentan estos niños con una frecuencia importante es la enfermedad de membrana hialina $(\mathrm{EMH})^{1}$, frecuente sobre todo en los recién nacidos de peso inferior a $1.000 \mathrm{~g}$, por la existencia de un pulmón muy inmaduro, con falta de desarrollo alveolar, escasas vías aéreas, abundante parénquima, arteriolas medias engrosadas y, sobre todo, por un déficit de agente tensioactivo pulmonar de la interfase aire-líquido alveolar, que conlleva un aumento de la tensión superficial del alvéolo con una tendencia al colapso al final de la espiración y una consecuente formación de atelectasia con alteración del patrón ventilación-perfusión.

De forma paralela al aumento de expectativa de vida en el conjunto de los grandes pretérminos, se plantea la cuestión de si esta expectativa también ha mejorado a lo largo de los años en los RNMBP con EMH. La disyuntiva es complicada porque, entre otras cosas, al entrar niños cada vez más pequeños en límites de viabilidad, la aparición de EMH va a ser más frecuente. Pero, por otro lado, las técnicas de ventilación mecánica y las terapias específicas para la prevención y tratamiento de este trastorno (administración de agente tensioactivo exógeno, de corticoides prenatales, etc.) han tratado de mejorar las expectativas de vida de estos niños.

La mayoría de los trabajos con agente tensioactivo en seres humanos, realizados después de haber sido probada su seguridad en animales de experimentación, datan de principios-mediados de los años noventa; fueron promovidos por firmas comerciales, para comprobar la utili- dad y seguridad del producto que trataban de introducir en la sintomatología. En ellos, solía administrarse agente tensioactivo a algunos niños y placebo a otros, respetando todos los condicionamientos éticos y jurídicos apropiados al efecto. En el momento de la realización de estos trabajos, como no se estaba seguro ni de la eficacia ni de la inocuidad de este tratamiento, esta actitud era moral y socialmente correcta. El haberse realizado en este caso un estudio transversal a lo largo del tiempo, incluso partiendo de épocas en las que no existían tratamientos específicos, ha hecho posible analizar el comportamiento en cuanto a la supervivencia en estos niños, sin la ayuda de determinados tratamientos que hoy día estamos obligados a administrar.

Con el objetivo de analizar los factores que influyen fundamentalmente en la mortalidad de estos niños, se ha recogido una muestra de 209 pacientes, de diferentes épocas, con pesos y edades gestacionales muy diversas, todos ellos con los diagnósticos RNMBP y EMH.

\section{Pacientes y métodos}

\section{Pacientes}

Se ha realizado un estudio retrospectivo con todos los recién nacidos que cumplían los criterios de inclusión de presentar un peso al nacimiento igual o inferior a $1.500 \mathrm{~g}$, tener enfermedad de membrana hialina, haber sido asistidos en el Servicio de Neonatología del Hospital Clínico San Carlos de Madrid en el período de tiempo comprendido entre el 1 de junio del año 1983 y el primero de enero de 1999 y no presentar malformaciones congénitas mayores. Representan un total de 211 recién nacidos de los cuales dos se excluyeron del análisis porque presentaron malformaciones congénitas mayores al nacimiento y fallecieron en las primeras horas de vida.

Las medidas generales aplicadas a los recién nacidos fueron las establecidas según los protocolos de Neonatología del Servicio en cada momento. La administración de agente tensioactivo exógeno sólo fue posible a partir del año 1992, en que se dispuso del mismo en este servicio. Hasta 1996 se utilizó fundamentalmente de forma "terapéutica" y posteriormente comenzó a introducirse en la práctica clínica de manera progresiva en forma "profiláctica", en niños menores de 28 semanas y de 1.250 g que comenzaban a necesitar fracción inspiratoria de oxígeno $\left(\mathrm{FiO}_{2}\right)>40$ para mantener $\mathrm{pH}$ y $\mathrm{pO}_{2}$ adecuados. La administración de corticoides prenatales también fue introduciéndose progresivamente en la clínica en la década de los años ochenta. Todas estas actuaciones se llevaron a cabo con previo consentimiento informado escrito de los padres.

Se administró un agente tensioactivo natural, extracto pulmonar bovino, Survanta ${ }^{\circledR}$ que era del que se disponía en el servicio; en ningún momento, ni hoy día, se considera que haya una clara superioridad de algún tipo de agente tensioactivo respecto a otro ${ }^{2-4}$. 
En relación con las características del paciente, se han definido las siguientes variables: "fecha de parto" distinguiendo a los nacidos antes de 1992 o a partir de este año; "enfermedad de membrana hialina", clasificada en grados según los hallazgos clínicos y radiológicos previos a la ventilación asistida; "sexo"; "peso al nacimiento" (g); "semanas de gestación"; "procedencia" (en el hospital mencionado o fuera del mismo); "administración de corticoides prenatales" (presente o ausente); "tipo de gestación" (simple o múltiple); "tipo de parto" (vaginal o por cesárea); "amniorrexis" (<24 ho $\geq 24$ h); "puntuación Apgar al primer y quinto minuto de vida; "administración de agente tensioactivo" (sí o no), "horas de vida en la administración de la primera dosis de surfactante" (no, profiláctico, terapéutico) y la existencia o no de "sepsis precoz" con confirmación bacteriológica.

La mortalidad se definió hasta el año de vida, con un seguimiento de los pacientes al alta.

\section{Método estadístico}

Para la identificación de los factores anteriormente descritos con significación estadística en la mortalidad de los recién nacidos de bajo peso con membrana hialina se ha utilizado el método de la regresión logística múltiple. El modelo de regresión logística permite además predecir la probabilidad de mortalidad en función de las variables descritas anteriormente 5 .

La forma de encontrar el modelo se basa en la metodología sugerida por Hosmer y Lemeshow ${ }^{5}$. Brevemente, este método consiste en incluir en el modelo aquellas variables que en el modelo univariante tienen una $p<0,25 y$ aquellas variables clínicamente importantes. Después estas variables deben incluirse en un modelo multivariante donde podrán ser eliminadas una vez que se evalúe su importancia conjuntamente $(\mathrm{p}<0,05)$. Una vez seleccionadas las variables del modelo se estudia la posibilidad de la no linealidad de las variables continuas y, por último, la posible interacción de las variables seleccionadas.

Para medir la bondad del ajuste utilizamos el test de Hosmer-Lemeshow y la curva ROC (receiver operator characteristic). Los análisis estadísticos se han realizado con el paquete estadístico SPSS 11.5.

\section{Resultados}

Estudiando las variables de los 209 recién nacidos observamos que hay $120(57,42 \%)$ varones y 89 (42,58\%) mujeres. Nacieron en el Hospital Clínico de Madrid 157 pacientes $(75,12 \%)$; los demás ingresaron trasladados desde otros centros; 143 recién nacidos $(68,42 \%)$ nacieron de embarazos simples, el resto fueron el fruto de gestaciones múltiples. Mientras que en 99 el alumbramiento fue por vía vaginal $(47,37 \%)$, en 110 fue por cesárea $(52,63 \%)$. En 187 casos $(89,47 \%)$ la amniorrexis se produjo en las $24 \mathrm{~h}$ antes del parto, sólo en 21 casos el parto sucedió después de $24 \mathrm{~h}$ de la rotura de la bolsa. En un caso no se pudo obtener esta información. Diez pacientes presentaron sepsis en las primeras $72 \mathrm{~h}$ de vida $(4,79 \%)$.

El peso de los niños al nacimiento se encuentra en un intervalo entre 500 y $1.500 \mathrm{~g}$ con una media de 1.016,23 y una desviación estándar (DE) de 255,06. La edad de gestación en el momento del parto fue muy variable, con un intervalo de 22 a 37 semanas, una media de 28,55 semanas y una DE de 2,57 semanas. La puntuación del test de Apgar de estos pacientes en el primer minuto de vida fue de 1 a 9, con los siguientes valores de cuartiles: 2 para el primer cuartil (percentil 25), 5 para la mediana (percentil 50) y 6 para el tercer cuartil (percentil 75). Más amplio fue el intervalo de puntuación Apgar en el quinto minuto, que osciló entre valores de 1 y 10 , con valores 5 para el primer cuartil, 7 para la mediana y 8 para el tercer cuartil. Del total de la muestra, 86 pacientes $(41,15 \%)$ fallecieron.

En 86 casos $(40,29 \%)$ las madres habían recibido alguna pauta de corticoides, parcial o completa; los restantes 123 casos $(59,71 \%)$ no recibieron corticoides prenatalmente. Fueron tratados 89 niños con una o varias dosis de agente tensioactivo exógeno $(42,6 \%)$, mientras que 120 no recibieron ninguna dosis $(57,4 \%)$. De manera terapéutica lo recibieron 61 recién nacidos y profiláctica 28.

Cobra especial interés describir el peso y la edad gestacional antes y después de que se pudiera aplicar agente tensioactivo. Así, antes de 1992, se recogieron 93 casos (44,5\% de la muestra) con un peso medio de $1.060,86$ g (mediana 1.070,00) de los cuales el 11,82\% tenían un peso menor a $750 \mathrm{~g}$ y el $39,78 \%$ un peso inferior a 1.000 g. Si nos fijamos a partir de 1992 (116 recién nacidos), tenemos que su peso medio 980,44 g (mediana: $957,50)$ de los cuales el $24,13 \%$ tiene un peso menor de $750 \mathrm{~g}$ y el $53,4 \%$ un peso inferior a $1.000 \mathrm{~g}$. En cuanto a la edad gestacional, tenemos antes de 1992 que la media de las semanas de gestación es 28,97 (mediana: 29,00) de los cuales el 12,90\% tienen 26 semanas o menos y el 55,91 menos de 30 semanas de gestación. A partir de 1992 la media es de 28,21 (mediana: 28,00) de los cuales el $24,13 \%$ tiene 26 semanas o menos y el 66,37 menos de 30 semanas. En las tablas 1 y 2 se resume información sobre las variables cuantitativas con media y DE, mientras que para las cualitativas se da el porcentaje, dicha información aparece pormenorizada para vivos y muertos en períodos de tiempo.

La construcción del modelo múltiple de regresión logística se ha realizado comenzando con los ajustes univariantes, resultando con $\mathrm{p}<0,25$ las variables: "grado de membrana hialina", con dos categorías (1 o 2, 3 o 4) $(\mathrm{p}=0,000) ;$ "sexo" $(\mathrm{p}=0,060)$; "peso al nacimiento" $(\mathrm{p}=0,000)$; "semanas de gestación" $(\mathrm{p}=0,000)$; "procedencia" ( $p=0,242)$; “ corticoides prenatales" con dos categorías (sí frente a no) ( $\mathrm{p}=0,009)$; "tipo de parto" $(\mathrm{p}=0,020)$; "puntuación Apgar al primer minuto de vida" $(\mathrm{p}=0,000) ; \mathrm{y}$ "puntuación Apgar al quinto minuto de 


\begin{tabular}{|c|c|c|c|}
\hline Factor & Período & Vivos & Fallecidos \\
\hline \multirow[t]{3}{*}{ Peso } & $<92$ & $1.127,4 \pm 190,6$ & $973,4 \pm 244,2$ \\
\hline & $\geq 92$ & $1.086,3 \pm 248,9$ & $803,1 \pm 201,5$ \\
\hline & Total & $1.103,4 \pm 226,5$ & $885,2 \pm 237,7$ \\
\hline \multirow[t]{3}{*}{ Edad gestacional } & $<92$ & $29,4 \pm 2,2$ & $28,4 \pm 2,8$ \\
\hline & $\geq 92$ & $29,2 \pm 2,1$ & $26,6 \pm 2,2$ \\
\hline & Total & $29,3 \pm 2,2$ & $27,5 \pm 2,7$ \\
\hline \multirow[t]{3}{*}{ Apgar al primer minuto } & $<92$ & $3,5,6$ & $2,5,6$ \\
\hline & $\geq 92$ & $3,5,6$ & $2,4,6$ \\
\hline & Total & $3,5,7$ & $2,4,5$ \\
\hline \multirow[t]{3}{*}{ Apgar a los $5 \mathrm{~min}$. } & $<92$ & $6,7,8$ & $5,7,8$ \\
\hline & $\geq 92$ & $5,7,8$ & $4,6,8$ \\
\hline & Total & $6,7,8$ & $4,6,7$ \\
\hline
\end{tabular}

TABLA 2. Porcentajes de las variables, según mortalidad y período de tiempo

\begin{tabular}{|c|c|c|c|}
\hline Factor & Período & $\begin{array}{c}\text { Vivos } \\
(\%)\end{array}$ & $\begin{array}{c}\text { Fallecidos } \\
(\%)\end{array}$ \\
\hline \multirow[t]{3}{*}{ Varones } & $<92$ & 57,7 & 61,0 \\
\hline & $\geq 92$ & 47,9 & 68,9 \\
\hline & Total & 52,0 & 65,1 \\
\hline \multirow[t]{3}{*}{ Nacidos en el Hospital Clínico } & $<92$ & 75,0 & 75,6 \\
\hline & $\geq 92$ & 80,3 & 66,7 \\
\hline & Total & 78,0 & 70,9 \\
\hline \multirow[t]{3}{*}{ Cesárea } & $<92$ & 53,8 & 34,1 \\
\hline & $\geq 92$ & 63,4 & 51,1 \\
\hline & Total & 59,3 & 43,0 \\
\hline \multirow[t]{3}{*}{ Grado de membrana hialina 3 o 4} & $<92$ & 23,1 & 78,05 \\
\hline & $\geq 92$ & 60,6 & 88,9 \\
\hline & Total & 44,7 & 83,7 \\
\hline \multirow[t]{3}{*}{$>1$ recién nacido } & $<92$ & 40,4 & 34,1 \\
\hline & $\geq 92$ & 28,2 & 24,4 \\
\hline & Total & 33,3 & 29,1 \\
\hline \multirow[t]{3}{*}{ Existencia de sepsis } & $<92$ & 3,8 & 4,9 \\
\hline & $\geq 92$ & 2,8 & 8,9 \\
\hline & Total & 3,3 & 7,0 \\
\hline \multirow[t]{3}{*}{ Amniorrexis $\geq 24 \mathrm{~h}$} & $<92$ & 9,6 & 17,5 \\
\hline & $\geq 92$ & 9,9 & 4,4 \\
\hline & Total & 9,8 & 10,6 \\
\hline Recibieron corticoides ${ }^{*}$ & $\geq 92$ & 50,7 & 40,0 \\
\hline Recibieron agente tensioactivo* & $\geq 92$ & 71,8 & 84,4 \\
\hline
\end{tabular}

*Sólo se dan resultados desde su aplicación a partir de 1992.

vida" ( $\mathrm{p}=0,000)$; y la existencia o no de "sepsis precoz" con confirmación bacteriológica $(p=0,225)$. Por ser una variable clínicamente importante "tratamiento con agente tensioactivo", con dos categorías (sí frente a no), se ha mantenido como posible candidata a entrar en el modelo multivariante a pesar de no ser significativa en el univariante $(p=0,6953)$, como sugiere la metodología de

\begin{tabular}{|c|c|c|c|}
\hline Variable & Coeficiente & $\mathrm{p}$ & OR \\
\hline Peso & $-0,0041$ & 0,0000 & 0,9959 \\
\hline Apgar a los $5 \mathrm{~min}$ & $-0,2201$ & 0,0166 & 0,8025 \\
\hline Corticoides* & $-0,8640$ & 0,0240 & 0,4215 \\
\hline Agente tensioactivo (sí) ${ }^{* *}$ & $-1,1858$ & 0,0050 & 0,3055 \\
\hline Grado de membrana hialina ( 3 o 4 ) & 2,0779 & 0,0000 & 7,9876 \\
\hline Constante & 4,6451 & & \\
\hline
\end{tabular}

* La administración de corticoides prenatales fue llevada a cabo **La administración de agente tensioactivo fue llevada a cabo. OR: odds ratio.

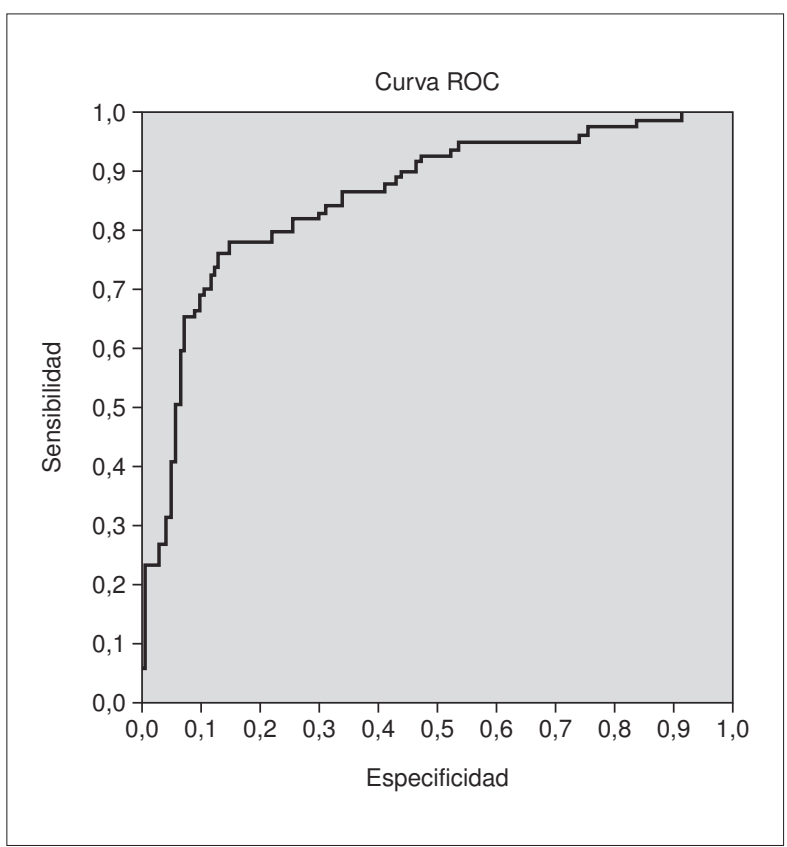

Figura 1. Curva ROC para el modelo de tabla 3.

Hosmer y Lemeshow ${ }^{5}$, la cual nos lleva al modelo final ajustado que se da en la tabla 3, donde sólo se seleccionan cinco variables: peso al nacimiento (peso), puntuación de Apgar al quinto minuto de vida (ag5), corticoides prenatales (cort), grado de membrana hialina $(\mathrm{g})$, tratamiento con agente tensioactivo (sur).

Si se observa la tabla 3 se aprecia que la probabilidad de fallecer disminuye con el aumento del peso, con el aumento de la puntuación Apgar al quinto minuto, la administración de corticoides prenatales y la administración de agente tensioactivo, aumentando dicha probabilidad con el aumento del grado de membrana hialina.

El estadístico del test de bondad de ajuste de Hosmer-Lemeshow para el modelo de la tabla 3 resulta 6,546 con $p=0,5863$, lo cual indica que este modelo se ajusta bien a los datos. El valor del área bajo la curva ROC (fig. 1) se evalúa mediante el estadístico $\mathrm{c}=0,858$, cuyo intervalo de confianza del $95 \%$ (IC $95 \%$ ) es $(0,805$, 
Figura 2. Probabilidad de fallecer para un recién nacido con membrana hialina con puntuación de Apgar al quinto minuto de 6 para una madre que ha sido tratada con corticoides, según va rían peso, grado de membrana ( $g r$ ) y tratamiento con agente tensioactivo (sío no).

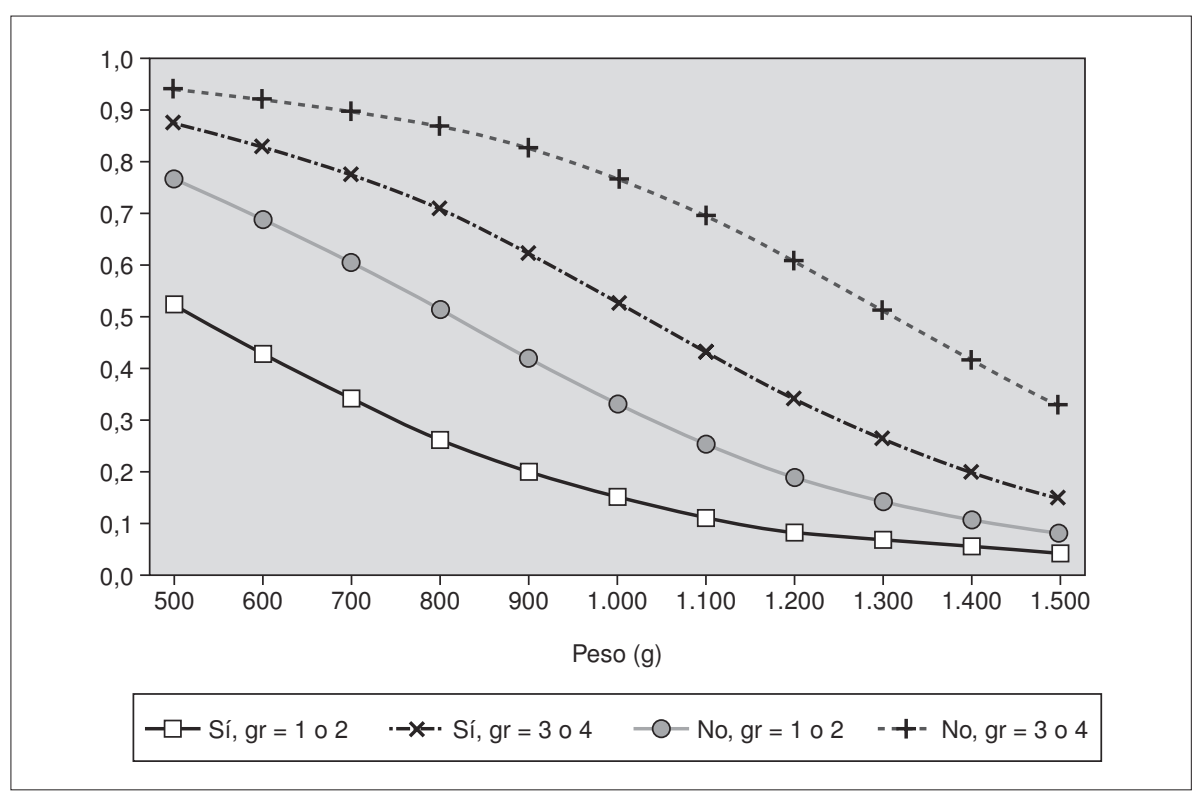

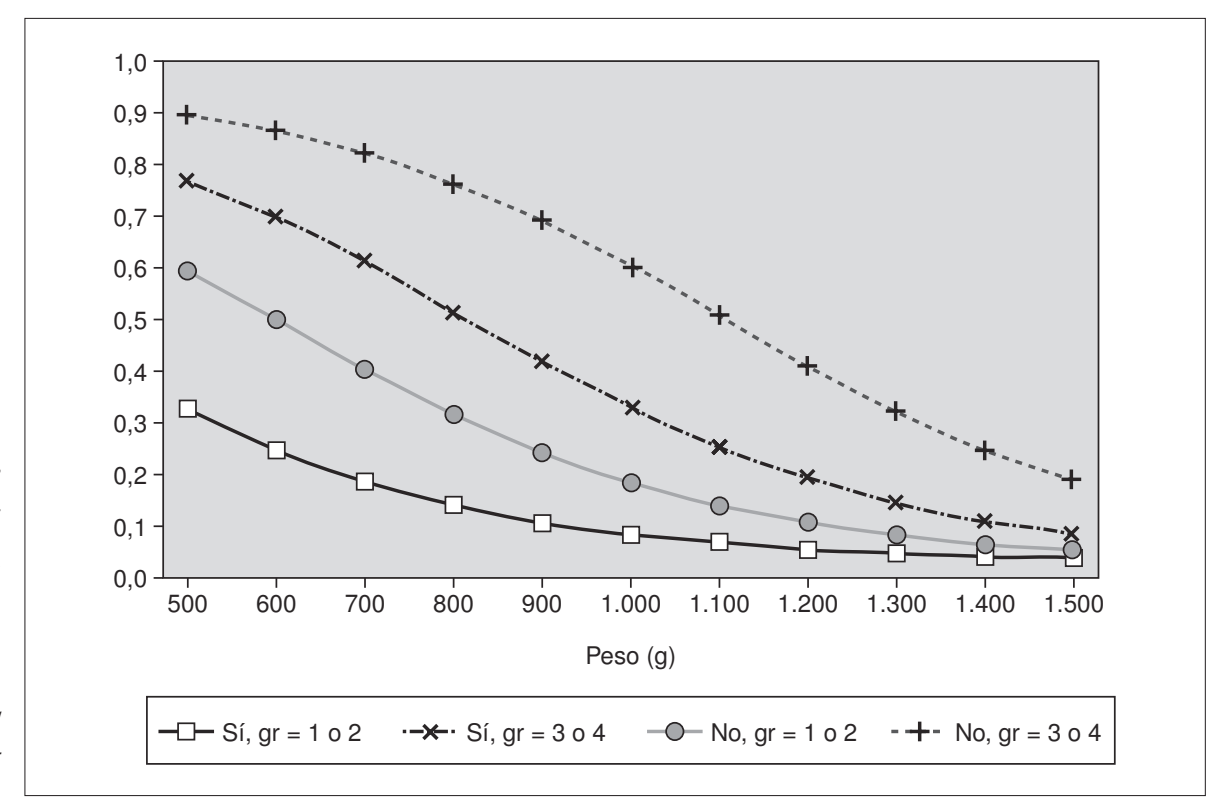

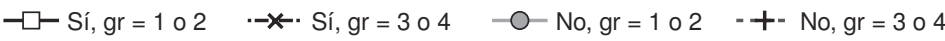

Figura 3. Probabilidad de mortalidad para un recién nacido con membrana hialina con puntuación de Apgar al quinto minuto de 6 para una madre que no ha sido tra tada con corticoides, según varían peso, grado de membrana (gr) y tratamiento con agente tensioactivo (sío no).
$0,912)$, lo cual indica también que este modelo es adecuado para predecir la probabilidad de morir de un recién nacido con membrana hialina.

Para valorar explícitamente de qué forma se modifica la probabilidad de mortalidad según varían las cinco variables del modelo de tabla 3 se dan, a modo de ejemplo, la figura 2 (dando corticoides a la madre) y la figura 3 ( $\sin$ dar corticoides a la madre) fijado un valor intermedio de Apgar 5. Observando cada una de estas dos figuras se puede ver cómo siempre tienen menor probabilidad de morir los niños a los que sí se les da agente tensioactivo frente que a los que no se les administra, también tienen menor probabilidad de morir los que tienen un grado de membrana (g) menor (1 o 2). La influencia del peso también que clara en cada figura. Si se observan conjuntamente las figuras 2 y 3 se puede ver cómo disminuye la probabilidad de morir al administrar corticoides.

\section{Discusión}

En las últimas décadas, el pronóstico de supervivencia de los grandes pretérminos con EMH ha mejorado sustancialmente. Se sigue profundizando en el conocimiento de los mecanismos fisiopatológicos que condicionan las afecciones de estos niños, lo cual conlleva la aplicación de unas medidas terapéuticas y unos cuidados que, al ser más adecuados, son diferentes a los aplicados en épo- 
cas pretéritas ${ }^{6}$. La introducción en la clínica de la terapia con agente tensioactivo parece haber supuesto un beneficio para estos pacientes, así como la terapia con corticoides prenatales ${ }^{7-14}$.

A pesar de la afluencia en la literatura especializada de numerosas publicaciones respecto a la administración de agente tensioactivo exógeno, existen pocos trabajos planteados de una forma similar a la que aquí se presenta, ya que en realidad existen pocas publicaciones científicas que analicen las épocas pre y postagente tensioactivo ${ }^{15}$. Además, en general los trabajos previamente realizados parten de muestras genéricas de niños pretérmino, mientras en nuestro trabajo se ha partido de una muestra de prematuros con EMH.

Ha habido mucha discusión en la literatura médica sobre el mejor momento para la aplicación de agente tensioactivo, de forma "profiláctica" (en las primeras $2 \mathrm{~h}$ de vida), o terapéutica (con el síndrome de dificultad respiratoria establecido $)^{16-20}$. En nuestro caso, la administración del mismo se realizó según las circunstancias y los protocolos ajustados en cada momento; por este motivo, es lógico que al hacer el análisis estadístico no se hayan apreciado diferencias en la evolución de los pacientes según el momento de administración del agente tensioactivo.

Gran parte de los investigadores que han intentado valorar los factores que intervienen en la mortalidad del RNMBP con $\mathrm{EMH}^{21}$ han procurado evitar la introducción de variables de confusión. No obstante, hay que tener en cuenta que con el paso del tiempo, por la experiencia acumulada, tanto a nivel asistencial como de investigación, se tiene un mejor cuidado y manejo de estos recién nacidos, lo que podría conllevar en general una disminución de la incidencia de fallecimientos. Prácticamente todos los autores están de acuerdo en que el peso y la edad gestacional influyen significativamente en esta mortalidad, esto concuerda con nuestros estudios univariantes. En el análisis multivariante realizado en este trabajo el peso se mantiene estadísticamente significativo, mientras que la importancia de la edad gestacional se diluye en presencia de las restantes variables. A la hora de predecir la mortalidad la información que aporta la edad gestacional se recoge en el resto de variables del modelo multivariante propuesto.

Existe un acuerdo generalizado, según los resultados de los trabajos publicados, en la reducción de la mortalidad con la terapia con agente tensioactivo, aunque no todos los autores han llegado a esta conclusión, lo que podría deberse a diferentes causas, como son tamaños muestrales insuficientes, existencia de factores de confusión o a falta de experiencia, sobre todo en los primeros años en que sólo se trataba a los más graves ${ }^{22}$. Los datos son críticos, en los niños de excesivo bajo peso, probablemente debido a que presentan una alta tasa de mortalidad por causas extrapulmonares.
Nuestros resultados coinciden con diversos trabajos publicados en la literatura especializada en cuanto al beneficio aditivo entre la terapia con agente tensioactivo y corticoides prenatales ${ }^{7-14}$, incluso cuando se estudian en el contexto de una metodología multifactorial, como ocurre en este trabajo en concreto, en el que además aparecen significativas otras variables mencionadas como el bajo peso al nacimiento, el test de Apgar bajo al quinto minuto y la gravedad de la EMH. En cambio, el test de Apgar al primer minuto no adquiere valor en el modelo de mortalidad final, lo que indica la importancia de la reanimación.

En algún trabajo el embarazo múltiple ${ }^{23}$ ha supuesto un impacto negativo en la supervivencia, variable no significativa en nuestro trabajo tal vez por no haber un número suficiente de embarazos múltiples en la muestra, o por el valor que cobran otras variables. Tampoco se ha singularizado en este estudio la importancia de la procedencia del recién nacido, nacido en el hospital o trasladado desde otro centro, aunque este factor sí parece cobrar su lógica importancia en la literatura médica, puesto que el transporte de un recién nacido, sobre todo con características como un muy bajo peso o con síndrome de dificultad respiratoria, parece empeorar la situación hemodinámica del mismo ${ }^{24}$. Hay que considerar que las causas de traslado no se consideraron en nuestro trabajo. Sería posible que algunos recién nacidos con extrema gravedad hubiesen fallecido en el traslado, antes de llegar al hospital, lo cual podría suponer un factor de confusión a la hora de analizar los datos en este punto en concreto.

El hecho de haber nacido por cesárea o parto vaginal tampoco ha destacado en el trabajo, aunque en otros trabajos realizados con recién nacidos algunos autores sí encuentran diferencias ${ }^{25,26}$ pero estos trabajan con poblaciones y metodologías distintas a las nuestras.

Podría recalcarse el hecho de que al haber podido ser realizado un estudio transversal a lo largo del tiempo, incluso partiendo de épocas en que no existían tratamientos específicos, ha concedido la oportunidad de analizar el comportamiento de determinadas enfermedades en los grandes pretérminos, con pesos muy bajos, sin la ayuda de estas terapias que hoy día no se podrían dejar de administrar. Sin embargo, es difícil valorar el papel específico de la importancia de cada terapia en concreto, puesto que hay cuidados que podrían modificar sustancialmente el pronóstico de estos pacientes.

Esta metodología de trabajo, basada en la selección de variables según Hosmer-Lemeshow, en la que se recomienda mantener como candidatas a entrar en el modelo multivariante las variables biológicamente significativas (aunque no lo sean estadísticamente en el análisis previo univariante), podría sentar las bases para una futura investigación de los aspectos relacionados con la morbilidad de estos grandes pretérminos con enfermedad de membrana hialina. 


\section{Biblografía}

1. Barton L, Hodgman JE, Pavlova Z. Causes of death in the extremely low birth weight infant. Pediatrics. 1999;103:446-51.

2. Soll RF. Natural surfactant extract versus synthetic surfactant for neonatal respiratory distress syndrome. Cochrane Database Syst Rev. 2000; CD000144.

3. Soll RF. Prophylactic natural surfactant extract for preventing morbidity and mortality in preterm infants (Cochrane Review). En: The Cochrane Library, 2, 2001; Oxford: Update Software.

4. Soll RF. Prophylactic synthetic surfactant for preventing morbidity and mortality in preterm infants (Cochrane Review). Cochrane Database Syst Rev. 2000;(2):CD000510.

5. Hosmer D, Lemeshow S. Applied logistic regression. John Wiley and Sons; 1989.

6. Harms K, Herting E, Kron M, Schill M, Schittmann H. Importance of pre and perinatal risk factors in respiratory distress syndrome of premature infants. A logical regression analysis of 1100 cases. Z Geburtshilfe Neonatol. 1997;201:258-62.

7. Crowley P. Prophylactic corticosteroids for preterm birth (Cochrane Review). En: The Cochrane Library, 2, 2001. Oxford: Update Software.

8. Crowley P. Prophylactic corticosteroids for preterm birth (Cochrane Review). En: The Cochrane Database Syst Rev. $2000 ;(2)$.

9. Modanlou HD, Beharry K, Padilla G, Iriye B. Combined effects of antenatal corticosteroids and surfactant supplementation on the outcome of very low birth weight infants. P Perinatol. 1996;16:422-8.

10. Rajagopal J, Kinane TB. Is there more to lung development than steroids and surfactant? Pediatrics. 2000;50:1103.

11. Sen S, Reghu A, Ferguson SD. Efficacy of a single dose of antenatal steroid in surfactant-treated babies 31 weeks' gestation. J Matern Fetal Neonatal Med. 2002;12:298-303.

12. Van Marter LJ, Allred EN, Levinton A, Pagano M, Parad R, Moore $\mathrm{M}$. Antenatal glucocorticoid treatment does not reduce chronic lung disease among surviving preterm infants. J Pediatr. 2001;138:198-204.

13. Walfisch A, Hallak M, Mazor M. Multiple courses of antenatal steroids: Risks and benefits. Obstet Gynecol. 2001;98:491-7.
14. Wijnberger LD, Mostert JM, Van Dam KI, Mol BW, Brouwers H, Visser GH. Comparison of single and repeated antenatal corticosteroid therapy to prevent neonatal death and morbidity in the preterm infant. Early Hum Dev. 2002;67:29-36.

15. Jacobs SE, O’Brien K, Inwood S, Kelly EN, Whyte HE. Outcome of infants 23-26 weeks' gestation pre and post surfactant. Acta Paediatr. 2000;89:959-65.

16. Morley CJ. Systematic review of prophylactic vs rescue surfactant. Arch Dis Child. 1997;77:F70-F4.

17. Osborn DA, Jeffery HE, Bredemeyer SL, Polverino JM, Reid S. Targeted early rescue surfactant in ventilated preterm infants using the click test. Pediatrics. 2000;106:E30.

18. Pandit PB, Dunn MS, Kelly EN, Periman N. Surfactant replacement in neonates with early chronic lung disease. Pediatrics. 1995;95:851-4.

19. Soll RF. Appropiate surfactant usage in 1996. Eur J Pediatr. 1996;155 Suppl 2P:8-13.

20. Yost CC, Soll RF. Early versus delayed selective surfactant treatment for neonatal respiratory distress syndrome. The Cochrane database of systemic review. Cochrane Database Syst Rev. 2000;(2):CD001456.

21. Chye JK, Lim CT. Very low birth weight enfants-mortality and predictive risk factors. Singapore Med J. 1999;40:565-70.

22. Hennes HM, Lee MB, Rimm AA, Shapiro DL Surfactant replacement therapy in respiratory distress syndrome. Meta-analysis of clinical trials of single-dose surfactant extracts. Am J Dis Child. 1992;146:534.

23. Nielsen HC, Harvey-Wilkes K, Mackinnon B, Hung S. Neonatal outcome of very premature infants from multiple and singleton gestations. Am J Obstet Gynecol. 1997;77:653-9.

24. Chien LY, Whyte R, Aziz K, Thiessen P, Matthew D, Lee SK. Canadian Neonatal Network: Improved outcome of preterm infants when delivered in tertiary care centers. Obstet Gynecol. 2001;98:247-52.

25. Hoop B, Kiwi R, Amini SB, Fanaroff A, Hack M. Neonatal morbidity after elective repeat cesarean section and trial of labor. Pediatrics. 1997;100:348-53.

26. Meirowitz NB, Ananth CV, Smulian JC, Vintzileos AM. Effect of labor on infant morbidity and mortality with preterm premature rupture of membranes: United States population-based study. Obstet Gynecol. 2001;97:494-8.

\section{Fe de errores}

En el artículo de J.A. Lepe Jiménez, A. Vázquez Florido, M. Ramos de Mora, A. Cervera Hidalgo, A. Rey Rodríguez y J. García Barrón titulado "Utilidad de la procalcitonina, proteína C reactiva y recuento leucocitario en la detección de enfermedad meningocócica durante un brote epidémico", publicado en An Pediatr (Barc). 2005;62:328-32, se ha detectado que los valores de proteína C reactiva se expresan en $\mathrm{ng} / \mathrm{ml}$ cuando lo correcto es expresarlos en $m g / l$. 